\title{
Drizzle: A Method for the Linear Reconstruction of Undersampled Images
}

\author{
A. S. Fruchter ${ }^{1}$ and R. N. Hook ${ }^{2}$ \\ ${ }^{1}$ Space Telescope Science Institute, 3700 San Martin Drive, Baltimore, MD 21218, USA; \\ fruchter@stsci.edu \\ ${ }^{2}$ Space Telescope European Coordinating Facility, Karl Schwarzschild Strasse 2, D-85748 \\ Garching, Germany; rhook@eso.org
}

\begin{abstract}
We have developed a method for the linear reconstruction of an image from undersampled, dithered data. The algorithm, known as Variable-Pixel Linear Reconstruction, or informally as "Drizzle", preserves photometry and resolution, can weight input images according to the statistical significance of each pixel, and removes the effects of geometric distortion both on image shape and photometry. This paper presents the method and its implementation. The photometric and astrometric accuracy and image fidelity of the algorithm as well as the noise characteristics of output images are discussed. In addition, we describe the use of drizzling to combine dithered images in the presence of cosmic rays.
\end{abstract}

\section{INTRODUCTION}

Undersampled images are common in astronomy, because instrument designers are frequently forced to choose between properly sampling a small field-of-view, or undersampling a larger field. Nowhere is this problem more acute than on the Hubble Space Telescope, whose corrected optics now provide superb resolution; however, the detectors on HST are only able to take full advantage of the full resolving power of the telescope over a limited field of view. For instance, the primary optical imaging camera on the HST, the Wide Field and Planetary Camera 2 (Trauger et al. 1994), is composed of four separate 800x800 pixel CCD cameras, one of which, the planetary camera (PC) has a scale of $0^{\prime \prime} 046$ per pixel, while the other three, arranged in a chevron around the PC, have a scale of $0{ }^{\prime \prime} 097$ per pixel. These latter three cameras, referred to as the wide field cameras (WFs), are currently the primary workhorses for deep imaging surveys on HST. However, these cameras greatly undersample the HST image. The width of a WF pixel equals the full-width at half-maximum (FWHM) of the optics in the the near-infrared, and greatly exceeds it in 
the blue. In contrast, a well-sampled detector would have $\gtrsim 2.5$ pixels across the FWHM. Other HST cameras such as NICMOS, STIS and the future Advanced Camera for Surveys (ACS) also suffer from undersampling to varying degrees. The effect of undersampling on WF images is illustrated by the "Great Eye Chart in the Sky" in Figure 1. Further examples showing astronomical targets are given in Section 8.

When the true distribution of light on the sky $T$ is observed by a telescope it is convolved by the point-spread function of the optics $O$ to produce an observed image, $I_{o}=T \otimes O$, where $\otimes$ represents the convolution operator. This effect is shown for the HST and WFPC2 optics by the upper-right panel in Figure 1. Pixelated detectors then again convolve this image with the response function of the electronic pixel $E$, thus $I_{d}=T \otimes O \otimes E$. The detected image can be thought of as this continuous convolved image sampled at the center of each physical pixel. Thus a shift in the position of the detector (know as a "dither") can be thought of as producing offset samples from the same convolved image. Although pixels are typically square on the detector, their response may be non-uniform, and indeed, may, because of the scattering of light and charge carriers, effectively extend beyond the physical pixel boundaries. This is the case in WFPC2. By contrast, in the NICMOS detectors (Storrs et al. 1999; Lauer 1999), the electronic pixel is effectively smaller than the physical pixel.

Fortunately, much of the information lost to undersampling can be restored. In the lower right of Figure 1 we display an image made using one of the family of techniques we refer to as "linear reconstruction." The most commonly used of these techniques are shift-and-add and interlacing. In interlacing, the pixels from the independent images are placed in alternating pixels on the output image according to the alignment of the pixel centers in the original images. The image in the lower right corner of Figure 1 has been restored by interlacing dithered images. However, due to the occasional small positioning errors of the telescope and the non-uniform shifts in pixel space caused by the geometric distortion of the optics, true interlacing of images is often infeasible. In the other standard linear reconstruction technique, shift-and-add, a pixel is shifted to the appropriate location and then added onto a sub-sampled image.

Shift-and-add can easily handle arbitrary dither postions, but it convolves the image yet again with the original pixel, compounding the blurring of the image and the correlation of the noise. In this case, two further convolutions are involved. The image is convolved with the physical pixel $P$, as this pixel is mathematically shifted over and added to the final image. In addition, when many images with different pointings are added together on the final output grid, there is also a convolution by the pixel of the final output grid $G$. This 


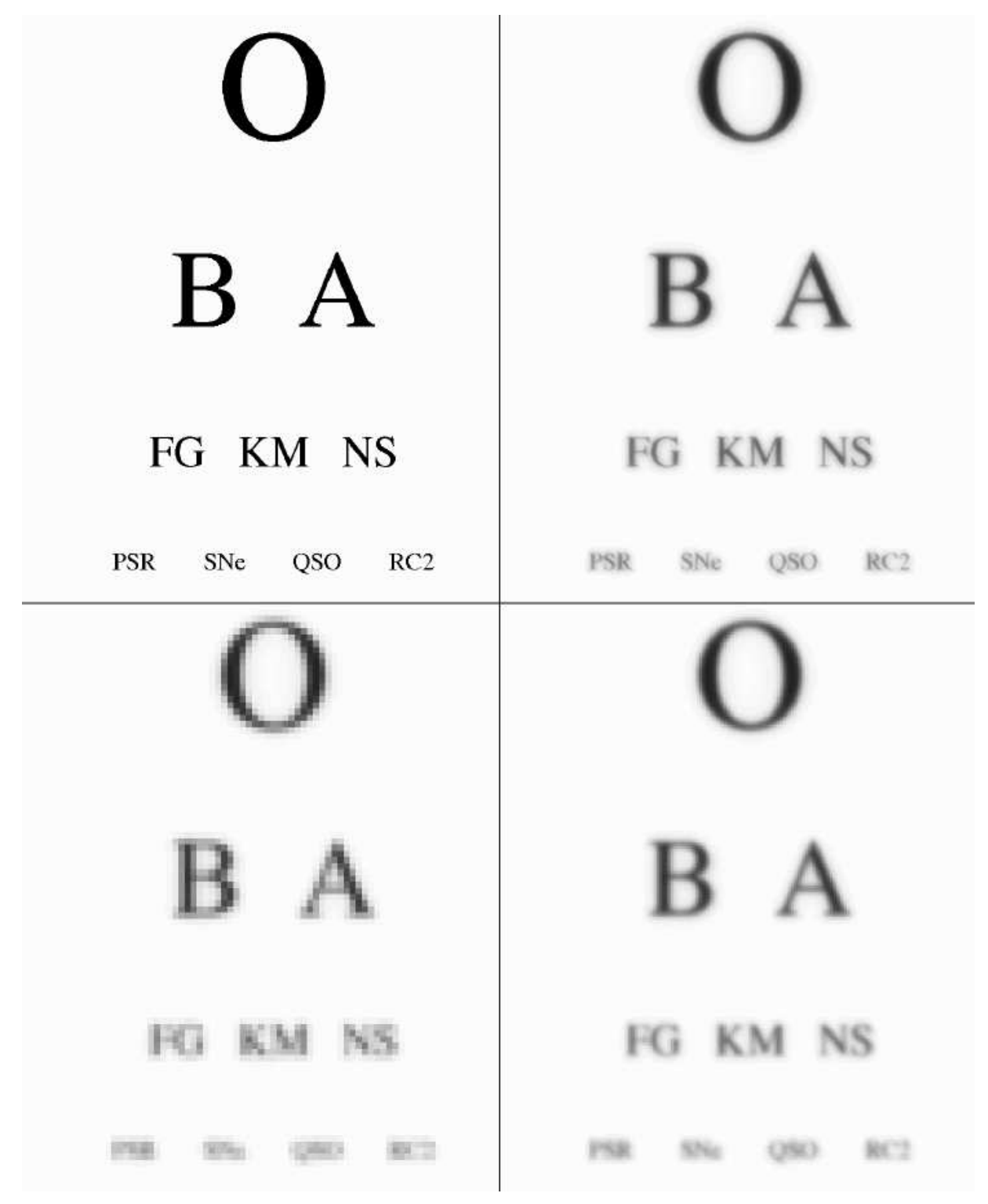

Fig. 1.- In the upper left corner of this figure, we present the "true image", i.e. the image one would see with an infinitely large telescope. The upper right shows the image after convolution with the optics of the Hubble Space Telescope and the WFPC2 camera - the primary wide-field imaging instrument presently installed on the HST. The lower left shows the image after sampling by the WFPC2 CCD, and the lower right shows a linear reconstruction of dithered CCD images. 
produces a final image

$$
I=T \otimes O \otimes E \otimes P \otimes G .
$$

The last convolution rarely produces a significant effect, however, as the output grid is usually considerably finer than the detector pixel grid, and convolutions add roughly as a sum of squares (the summation is exact if the convolving functions are Gaussians).

The importance of avoiding convolutions by the detector pixel is emphasized by comparing the upper and lower right hand images in Figure 1. The deterioration in image quality between these images is due entirely to the single convolution of the image by the WF pixel. The interlaced image in the lower-right panel has had the sampled values from all of the input images directly placed in the appropriate output pixels without further convolution by either $P$ or $G$.

Here we present a new method, Drizzle, which was originally developed for combining the dithered images of the Hubble Deep Field North (HDF-N) (Williams et al. 1996) and has since been widely used both for the combination of dithered images from HST's cameras and those on other telescopes. Drizzle has the versatility of shift-and-add yet largely maintains the resolution and independent noise statistics of interlacing. While other methods (c.f. Lauer 1999) have been suggested for the linear combination of dithered images, Drizzle has the advantage of being able to handle images with essentially arbitrary shifts, rotations and geometric distortion, and, when given input images with proper associated weight maps, creates an optimal statistically summed image. Drizzle also naturally handles images with "missing" data, due, for instance, to corruption by cosmic rays or detector defects.

The reader should note that Drizzle does not attempt to improve upon the final image resolution by enhancing the high frequency components of the image which have been suppressed either by the optics or the detector. While such procedures, which we refer to as "image restoration" (in contrast to "image reconstruction"), are frequently very valuable (see Hanisch and White 1994 for a review), they invariably trade signal-to-noise for enhanced resolution. Drizzle, on the other hand, was developed specifically to provide a flexible and general method of image combination which produces high resolution results without sacrificing the final signal-to-noise.

\section{THE METHOD}

Although the effect of Drizzle on the quality of the image can be profound, the algorithm is conceptually straightforward. Pixels in the original input images are mapped 
into pixels in the subsampled output image, taking into account shifts and rotations between images and the optical distortion of the camera. However, in order to avoid re-convolving the image with the large pixel "footprint" of the camera, we allow the user to shrink the pixel before it is averaged into the output image, as shown in Figure 2.

The new shrunken pixels, or "drops", rain down upon the subsampled output. In the case of the HDF-N WFPC2 imaging, the drops were given linear dimensions one-half that of the input pixel - slightly larger than the dimensions of the output pixels. The value of an input pixel is averaged into an output pixel with a weight proportional to the area of overlap between the "drop" and the output pixel. Note that if the drop size is sufficiently small not all output pixels have data added to them from each input image. One must therefore choose a drop size that is small enough to avoid degrading the image, but large enough so that after all images are drizzled the coverage is reasonably uniform.

The drop size is controlled by a user-adjustable parameter called pixfrac, which is simply the ratio of the linear size of the drop to the input pixel (before any adjustment due to the geometric distortion of the camera). Thus interlacing is equivalent to Drizzle in the limit of pixfrac $\rightarrow 0.0$, while shift-and-add is equivalent to pixfrac $=1.0$. The degree of subsampling of the output is controlled by the user through the scale parameter $s$, which is the ratio of the linear size of an output pixel to an input pixel.

When a pixel $\left(x_{i}, y_{i}\right)$ from an input image $i$ with data value $d_{x_{i} y_{i}}$ and user defined weight $w_{x_{i} y_{i}}$ is added to an output image pixel $\left(x_{o}, y_{o}\right)$ with value $I_{x_{o} y_{o}}$, weight $W_{x_{o} y_{o}}$, and fractional pixel overlap $0<a_{x_{i} y_{i} x_{o} y_{o}}<1$, the resulting values and weights of that same pixel, $I^{\prime}$ and $W^{\prime}$ are

$$
\begin{aligned}
W_{x_{o} y_{o}}^{\prime} & =a_{x_{i} y_{i} x_{o} y_{o}} w_{x_{i} y_{i}}+W_{x_{o} y_{o}} \\
I_{x_{o} y_{o}}^{\prime} & =\frac{d_{x_{i} y_{i}} a_{x_{i} y_{i} x_{o} y_{o}} w_{x_{i} y_{i}} s^{2}+I_{x_{o} y_{o}} W_{x_{o} y_{o}}}{W_{x_{o} y_{o}}^{\prime}}
\end{aligned}
$$

where a factor of $s^{2}$ is introduced to conserve surface intensity, and where $i$ and $o$ are used to distinguish the input and output pixel indices. In practice, Drizzle applies this iterative procedure to the input data, pixel-by-pixel, image-by-image. Thus, after each input image is processed, there is a usable output image and weight, $I$ and $W$.

The final output images, after all inputs have been processed, can be written as

$$
\begin{aligned}
W_{x_{o} y_{o}} & =a_{x_{i} y_{i} x_{o} y_{o}} w_{x_{i} y_{i}} \\
I_{x_{o} y_{o}} & =\frac{d_{x_{i} y_{i}} a_{x_{i} y_{i} x_{o} y_{o}} w_{x_{i} y_{i}} s^{2}}{W_{x_{o} y_{o}}}
\end{aligned}
$$

where for these Equations, 4 and 5, we use the Einstein convention of summation over repeated indices, and where the input indices $x_{i}$ and $y_{i}$ extend over all input images. It 


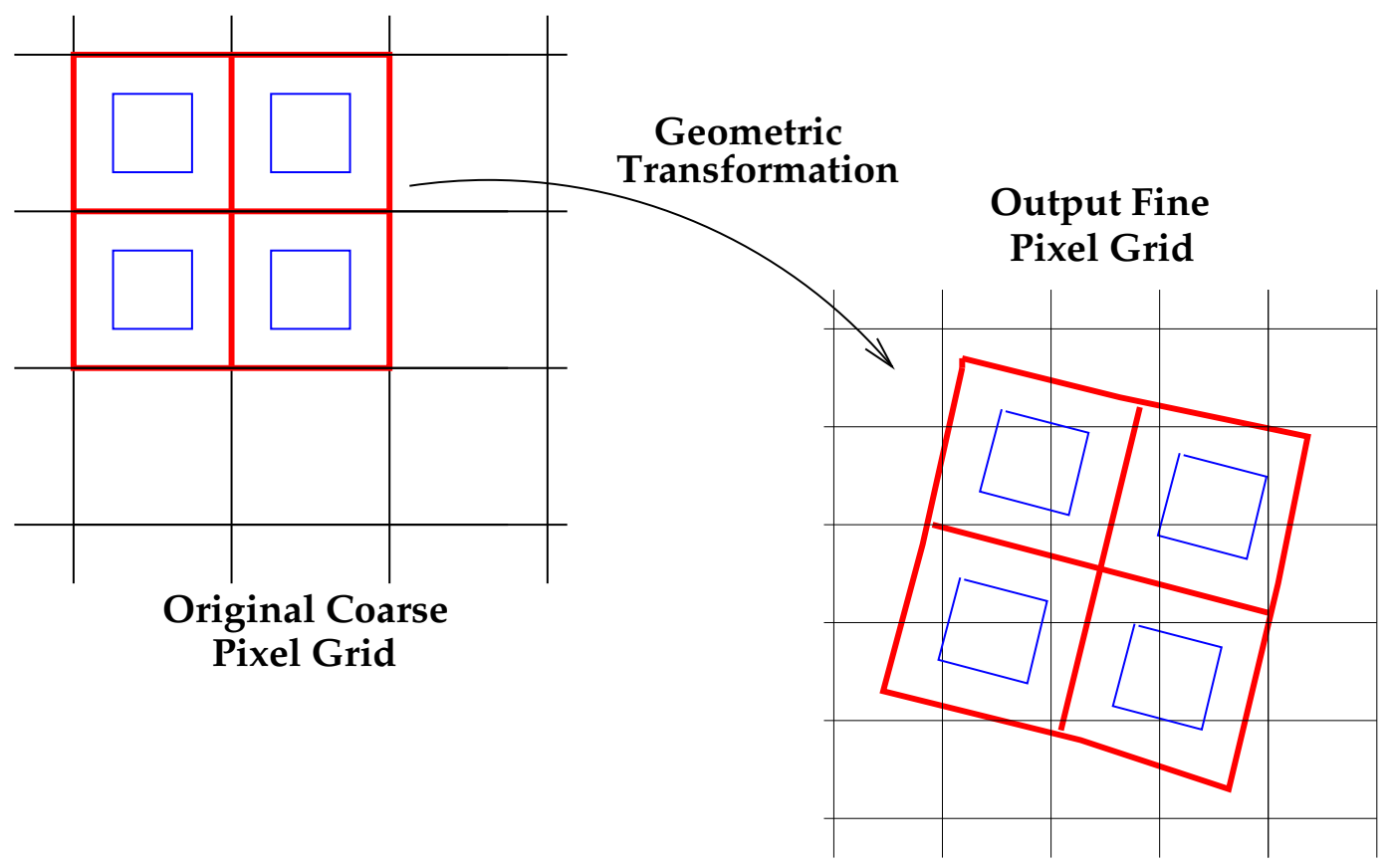

Fig. 2.- A schematic representation of Drizzle. The input pixel grid (shown on the left) is mapped onto a finer output grid (shown on right), taking into accounts shift, rotation and geometric distortion. The user is allowed to "shrink" the input pixels to smaller pixels, which we refer to as drops (faint inner squares). A given input image only affects output image pixels under drops. In this particular case, the central output pixel receives no information from the input image. 
is worth noting that in nearly all cases $a_{x_{i} y_{i} x_{o} y_{o}}=0$, since very few input pixels overlap a given output pixel.

This algorithm has a number of advantages over the more standard linear reconstruction methods presently used. It preserves both absolute surface and point source photometry (though see Section 5 for a more detailed discussion of point source photometry). Therefore flux can be measured using an aperture whose size is independent of position on the chip. And as the method anticipates that a given output pixel may receive no information from

a given input pixel, missing data (due for instance to cosmic rays or detector defects) does not cause a substantial problem, so long as there are enough dithered images to fill in the gaps caused by these zero-weight input pixels. Finally, the linear weighting scheme is statistically optimum when inverse variance maps are used as the input weights.

Drizzle replaces the convolution by $P$ in Equation 1 with a convolution with $p$, the pixfrac. As this kernel is usually smaller the full pixel, and as noted earlier convolutions add as the sum of squares, the effect of this replacement is often quite significant. Furthermore, when the dithered positions of the input images map directly onto the centers of the output grid, and pixfrac and scale are chosen so that $p$ is only slightly greater than $s$, one obtains the full advantages of interlacing: because the power in an output pixel is almost entirely determined by input pixels centered on that output pixel, the convolutions with both $p$ and $G$ effectively drop away. Nonetheless, the small overlap between adjacent drops fills in missing data.

\section{COSMIC RAY DETECTION}

Few HST observing proposals have sufficient time to take a number of exposures at each of several dither positions. Therefore, if dithering is to be of wide-spread use, one must be able to remove cosmic rays from data where few, if any, images are taken at the same position on the sky. We have therefore adapted Drizzle to the removal of cosmic rays. As the techniques involved in cosmic ray removal are also valuable in characterizing the image fidelity of Drizzle, we will discuss them first.

Here then is short description of the method we use for the removal of cosmic rays:

1. Drizzle each image onto a separate sub-sampled output image using pixfrac $=1.0$.

2. Take the median of the resulting aligned drizzled images. This provides a first estimate of an image free of cosmic-rays. 
3. Map the median image back to the input plane of each of the individual images, taking into account the image shifts and geometric distortion. This can done by interpolating the values of the median image using a program we have named "Blot".

4. Take the spatial derivative of each of the blotted output images. This derivative image is used in the next step to estimate the degree to which errors in the computed image shift or the blurring effect of taking the median could have distorted the value of the blotted estimate.

5. Compare each original image with the corresponding blotted image. Where the difference is larger than can be explained by noise statistics, the flattening effect of taking the median or an error in the shift, the suspect pixel is masked.

6. Repeat the previous step on pixels adjacent to pixels already masked, using a more stringent comparison criterion.

7. Finally, drizzle the input images onto a single output image using the pixel masks created in the previous steps. For this final combination a smaller pixfrac than in step 1) will usually be used in order to maximize the resolution of the final image.

Figure 3 shows the result of applying this method to data originally taken by Cowie and colleagues (Cowie, Hu, and Songaila 1995). The reduction was done using a set of IRAF (Tody 1993) scripts which are now available along with Drizzle in the Dither package of STSDAS. In addition to demonstrating how effectively cosmic rays can be removed from singly dithered images (i.e. images which share no common pointing), this image also displays the degree to which linear reconstruction can improve the detail of an image. In the Drizzled image the object to the upper right clearly has a double nucleus (or a single nucleus with a dust lane through it), but in the original image the object appears unresolved.

\section{IMAGE FIDELITY}

The drizzling algorithm was designed to obtain optimal signal-to-noise on faint objects while preserving image resolution. These goals are unfortunately not fully compatible. As noted earlier, non-linear image restoration procedures which attempt to remove the blurring due to the PSF and the pixel response function through enhancing the high frequencies in the image, such as the Richardson-Lucy (Richardson 1972; Lucy 1974; Lucy and Hook 1991) and maximum entropy methods (Gull and Daniell 1978; Weir and Djorgovski 1990), 

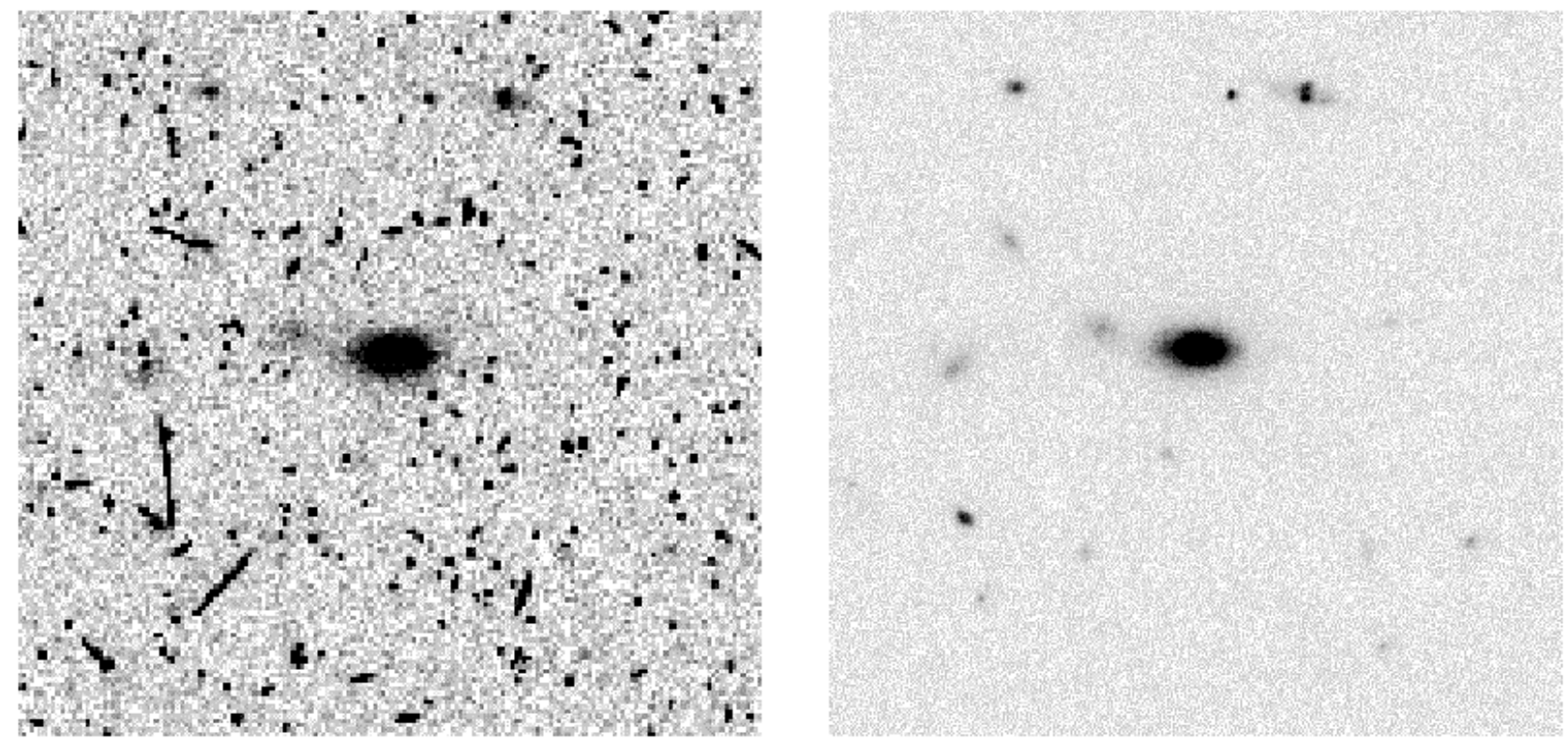

Fig. 3.- The image on the left shows a region of one of twelve 2400s archival images taken with the F814W wide near-infrared filter on WFPC2. Numerous cosmic rays are visible. On the right is the drizzled combination of the twelve images, no two of which shared a dither position.

directly exchange signal-to-noise for resolution. In the drizzling algorithm no compromises on signal-to-noise have been made; the weight of an input pixel in the final output image is entirely independent of its position on the chip. Therefore, if the dithered images do not uniformly sample the field, the "center of light" in an output pixel may be offset from the center of the pixel, and that offset may vary between adjacent pixels. Dithering offsets combined with geometric distortion generally produce a sampling pattern that varies across the field. The output PSFs produced by the combination of such irregularly dithered datasets may, on occasion, show variations about the true PSF. Fortunately this does not noticeably affect aperture photometry performed with typical aperture sizes. In practice the variability appears larger in WFPC2 data than we would predict based on our simulations. Examination of more recent dithered stellar fields leads us to suspect that this excess variability results from a problem with the original data, possibly caused by charge transfer errors in the CCD (Whitmore and Heyer 1997). 


\section{PHOTOMETRY}

Camera optics generally introduce geometric distortion of images. In the case of the WFPC2, pixels at the corner of each CCD subtend less area on the sky than those near the center. This effect will be even more pronounced in the case of the Advanced Camera for Surveys (ACS). However, after application of the flat field, a source of uniform surface brightness on the sky produces uniform counts across the CCD. Therefore, point sources near the corners of the chip are artificially brightened compared to those in the center. By scaling the weights of the input pixels by their areal overlap with the output pixel, and by moving input points to their corrected geometric positions, Drizzle largely removes this effect. In the case of pixfrac $=1$, this correction is exact.

In order to study the ability of Drizzle to remove the photometric effects of geometric distortion when pixfrac is not identically equal to one, we created a four times sub-sampled grid of $19 \times 19$ artificial stellar PSFs. This image was was then blotted onto four separate images, each with the original WF sampling, but dithered in a four-point pattern of half-pixel shifts. As a result of the geometric distortion of the WF camera, the stellar images in the corners of these images appear up to $\sim 4 \%$ brighter in the corners of the images than near the center. These images were then drizzled with a scale $=0.5$ and pixfrac $=0.6$. Aperture photometry on the $19 \times 19$ grid after drizzling reveals that the effect of geometric distortion on the photometry has been dramatically reduced: the RMS photometric variation in the drizzled image is 0.004 mags. Of course this is not the final photometric error of a drizzled image (which will depend on the quality of the input images), but only the additional error which the use of Drizzle would add under these rather optimal circumstances.

In practice users may not have four relatively well interlaced images but rather a number of almost random dithers, and each dithered image may suffer from cosmic ray hits. Therefore, in a separate simulation, we have used the shifts actually obtained in the WF2 F814W images of the HDF-N as an example of the nearly random sub-pixel phase that large dithers may produce on HST. In addition, we have associated with each image a mask corresponding to cosmic ray hits from one of the deep HST WF images used in creating Figure 3. When these simulated images are drizzled together, the root mean-square noise in the final photometry (which does not include any errors that could occur because of missed or incorrectly identified cosmic rays) is $\lesssim 0.015$ mags. Figure 4 displays the results of this process. 

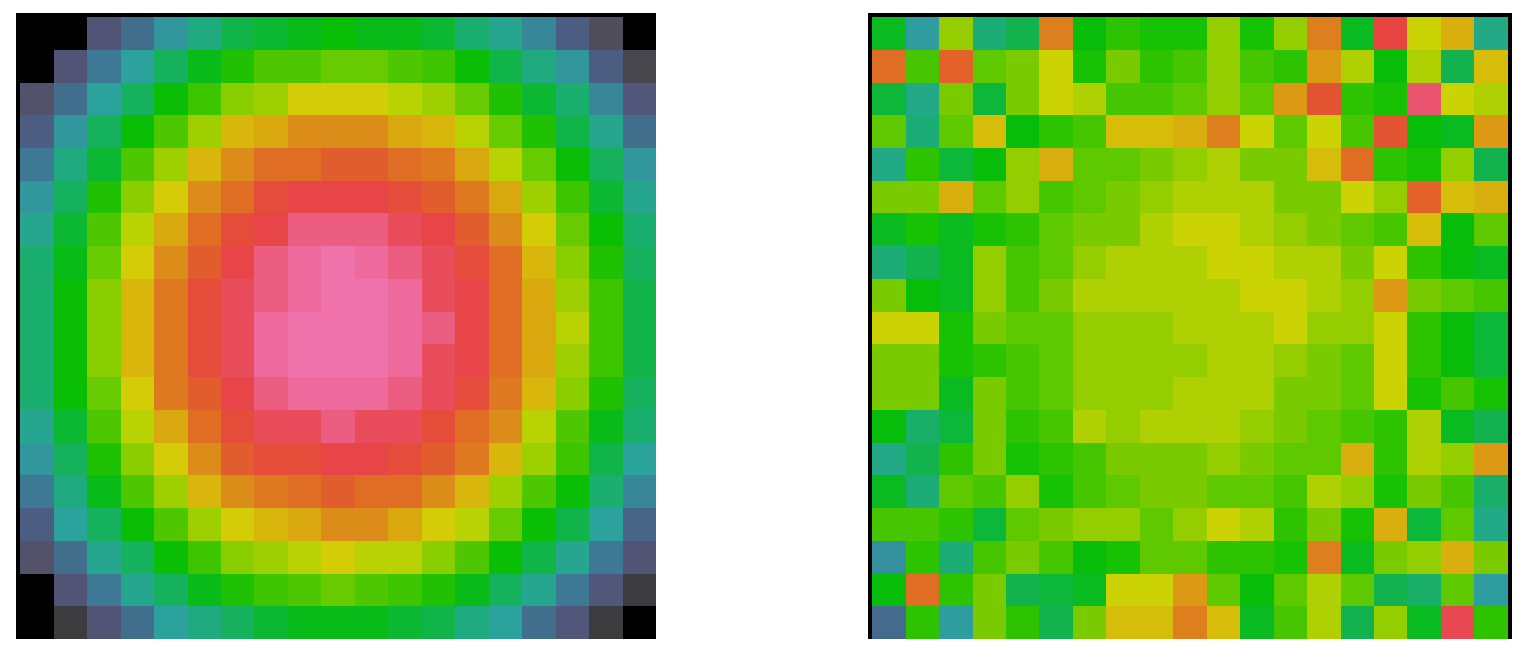

Fig. 4.- An example of the effect of geometric distortion on photometry. The pixels in the two images represent the photometric values of a $19 \times 19$ grid of stars on the WF chip. Each pixel represents the photometric value of a star; the pixels are not images of the stars. The figure on the left shows the apparent brightness of the stars (all of equal intrinsic brightness) as they would appear in a flat-fielded WF image. As pixels near the edge of the chip are up to $4 \%$ smaller on the sky than pixels near the center, the flat field has artificially brightened the stars near the edges and the corners. The image on the right shows the photometric values of these stars after drizzling, including the use of representative cosmic ray masks (see Figure 3). The standard deviation of the corrected stellar magnitudes $\leq 0.015$ mags. 


\section{ASTROMETRY}

We have also evaluated the effect of drizzling on astrometry. The stellar images described in the previous section were again drizzled using the HDF shifts as above, setting $\mathrm{scale}=0.5$ and pixfrac $=0.6$. Both uniform weight files and cosmic ray masks were used. The positions of the drizzled stellar images were then determined with the "imexam" task of IRAF, which locates the centroid using the marginal statistics of a box about the star. A box with side equal to 6 output pixels, or slightly larger than twice the full-width at half

maximum of the stellar images, was used. A root mean square scatter of the stellar positions of $\sim 0.018$ input pixels about the true position was found for the images created with uniform weight files and the cosmic-ray masks. However, we find an identical scatter when we down-sample the original four-times oversampled images to the two-times oversampled scale of the test images. Thus it appears that no additional measurable astrometric error has been introduced by Drizzle. Rather we are simply observing the limitations of our ability to centroid on images which contain power that is not fully Nyquist sampled even when using pixels half the original size.

\section{NOISE IN DRIZZLED IMAGES}

\subsection{The Nature of the Problem}

Drizzle frequently divides the power from a given input pixel between several output pixels. As a result, the noise in adjacent pixels will be correlated. Understanding this effect in a quantitative manner is essential for estimating the statistical errors when drizzled images are analysed using object detection and measurement programs such as SExtractor (Bertin and Arnouts 1996) and DAOPHOT (Stetson 1987).

The correlation of adjacent pixels implies that a measurement of the noise in a drizzled image on the output pixel scale underestimates the noise on larger scales. In particular, if one block sums a drizzled image by $\mathrm{N} \times \mathrm{N}$ pixels, even using a proper weighted sum of the pixels, the per-pixel noise in the block summed image will generally be more than a factor of $\mathrm{N}$ greater than the per-pixel noise of the original image. The factor by which the ratio of these noise values differs from $\mathrm{N}$ in the limit as $\mathrm{N} \Rightarrow \infty$ we refer to as the noise correlation ratio, $\mathcal{R}$.

One can easily see how this situation arises by examining Figure 5. In this figure we show an input pixel (broken up into two regions, a and b) being drizzled onto an output pixel plane. Let the noise in this pixel be $\epsilon$ and let the area of overlap of the drizzled pixel 


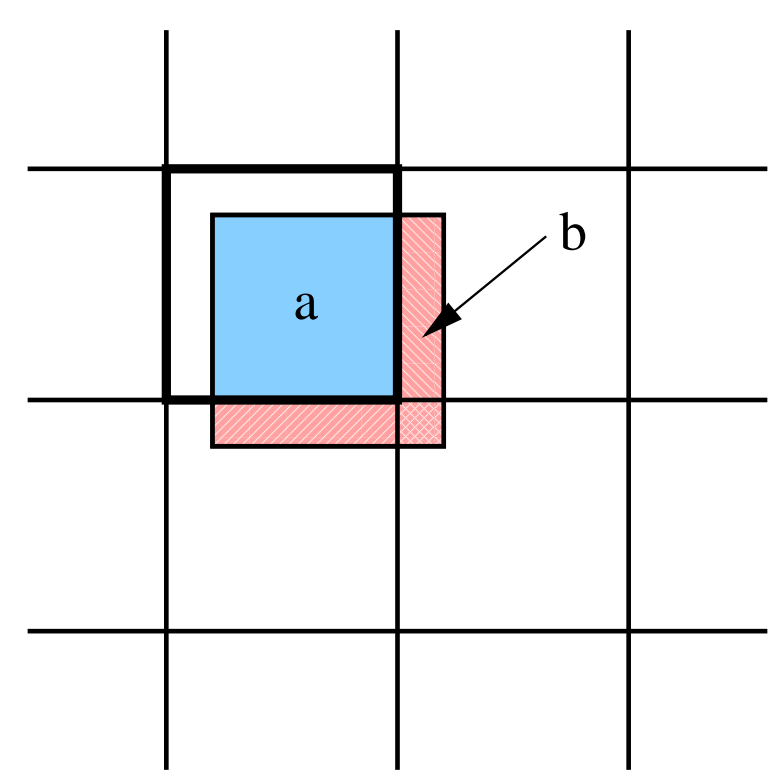

Fig. 5. - A schematic view of the distribution of noise from a single input between neighboring output pixels. A more complete description is provided in the body of the paper.

with the "primary" output pixel (shown with a heavier border) be $a$, and the areas of overlap with the other three pixels be $b_{1}, b_{2}$, and $b_{3}$, where $b=b_{1}+b_{2}+b_{3}$, and $a+b=1$. Now the total noise power added to the image variance is, of course, $\epsilon^{2}$; however, the noise that one would measure by simply adding up the variance of the output image pixel-by-pixel would be

$$
\left(a^{2}+b_{1}^{2}+b_{2}^{2}+b_{3}^{3}\right) \epsilon^{2}<\epsilon^{2}
$$

The inequality exists because all cross terms $\left(a b_{1}, a b_{2}, b_{1} b_{2} \ldots\right)$ are missed by summing the squares of the individual pixels. These terms, which represent the correlated noise in a drizzled image, can be significant.

\subsection{The Calculation}

In general, the correlation between pixels, and thus $\mathcal{R}$, depends on the choice of drizzle parameters and geometry and orientation of the dither pattern, and often varies across an image. While it is always possible to estimate $\mathcal{R}$ for a given set of drizzle parameters and dithers, in the case where all output pixels receive equivalent inputs (in both dither pattern and noise, though not necessarily from the same input images) the situation becomes far more analytically tractable. In this case, calculating the noise properties of a single pixel 
gives one the noise properties of the entire image.

Consider then the situation when pixfrac, $p$, is set to zero. There is then no correlated noise in the output image - since a given input pixel contributes only to the output pixel which lies under its center, and the noise in the individual input pixels is assumed to be independent. Let $d_{x y}$ represent a pixel from any of the input images, and let $\mathcal{C}$ be the set of all $d_{x y}$ whose centers fall on a given output pixel of interest. Then it is simple to show from Equations 4 and 5 that the expected variance of the noise in that output pixel, when $p=0$, is simply

$$
\sigma_{c}^{2}=\sum_{d_{x y} \in \mathcal{C}} w_{x y}^{2} s^{4} \sigma_{x y}^{2} /\left(\sum_{d_{x y} \in \mathcal{C}} w_{x y}\right)^{2}
$$

where $\sigma_{x y}$ is the standard deviation of the noise distribution of the input pixel $d_{x y}$. We term this $\sigma_{c}$, as it is the standard deviation calculated with the pixel values added only to the pixels on which they are centered.

Now let us consider a drizzled output image where $p>0$. In this case, the set of pixels contributing to an output pixel will not only include pixels whose centers fall on the output pixel, but also those for which a portion of the drop lands on the output pixel of interest even though the center does not. We refer to the set of all input pixels whose drops overlap with a given output pixel as $\mathcal{P}$ and note that $\mathcal{C} \subset \mathcal{P}$. The variance of the noise in a given output pixel is then

$$
\sigma_{p}^{2}=\sum_{d_{x y} \in \mathcal{P}} a_{x y}^{2} w_{x y}^{2} s^{4} \sigma_{x y}^{2} /\left(\sum_{d_{x y} \in \mathcal{P}} a_{x y} w_{x y}\right)^{2}
$$

where $a_{x y}$ is the fractional area overlap of the drop of input data pixel $d_{x y}$ with the output pixel $o$. Here we choose the symbol $\sigma_{p}$ to represent the standard deviation calculated from all pixels that contribute to the output pixel when pixfrac $=p$. The degree to which $\sigma_{p}^{2}$ and $\sigma_{c}^{2}$ differ depends on the dither pattern and the values of $p$ and $s$. However, as more input pixels are averaged together to estimate the value of a given output pixel in $\mathcal{P}$ than in $\mathcal{C}, \sigma_{p}^{2} \leq \sigma_{c}^{2}$. When $p=0, \sigma_{p}$ is by definition equal to $\sigma_{c}$.

Now consider the situation where we block average a region of $\mathrm{N} \times \mathrm{N}$ pixels of the final drizzled image, doing a proper weighted sum of the image pixels. This sum is equivalent to having drizzled onto an output image with a scale size $\mathrm{N} s$. But as $\mathrm{N} s \gg p$, this approaches the sum over $\mathrm{C}$, or, in the limit of large $N, N \sigma_{c}$. However, a prediction of the noise in this region, based solely on a measurement of the pixel-to-pixel noise, without taking into account the correlation between pixels would produce $N \sigma_{p}$. Thus we see that

$$
\mathcal{R}=\frac{\sigma_{c}}{\sigma_{p}}
$$


One can therefore obtain $\mathcal{R}$ for a given set of drizzle parameters and dither pattern by calculating $\sigma_{c}$ and $\sigma_{p}$ and performing the division. However, there is a further simplification that can be made. Because we have assumed that the inputs to each pixel are statistically equivalent, it follows that the weights of the individual output pixels in the final drizzled image are independent of the choice of $p$. To see this, notice that the total weight of a final image (that is the sum of the weights of all of the pixels in the final image) is independent of the choice of $p$. Ignoring edge pixels, the number of pixels in the final image with non-zero weight is also independent of the choice of $p$. Yet as the fraction of pixels within $p$ of the edge scales as $1 / \mathrm{N}$, and the weight of an interior pixel cannot depend on $\mathrm{N}$, we see that the weight of an interior pixel must also be independent of $p$. As a result $\sum_{d_{x y} \in \mathcal{C}} w_{x y}=\sum_{d_{x y} \in \mathcal{P}} a_{x y}^{2} w_{x y}$.

Therefore, we find that

$$
\mathcal{R}^{2}=\frac{\sigma_{c}^{2}}{\sigma_{p}^{2}}=\frac{\sum_{d_{x y} \in \mathcal{C}} w_{x y}^{2} \sigma_{x y}^{2}}{\sum_{d_{x y} \in \mathcal{P}} a_{x y}^{2} w_{x y}^{2} \sigma_{x y}^{2}}
$$

Although $\mathcal{R}$ must be calculated for any given set of dithers, there is perhaps one case that is particularly illustrative. When one has many dithers, and these dithers are fairly uniformly placed across the pixel, one can approximate the effect of the dither pattern on the noise by assuming that the dither pattern is entirely uniform and continuously fills the output plane. In this case the above sums become integrals over the output pixels, and thus it is not hard (though somewhat tedious) to derive $\mathcal{R}$. If one defines $r=p / s$ where $p=$ pixfrac and $s=$ scale, then in the case of a filled uniform dither pattern one finds,

$$
\text { if } r \geq 1
$$

$$
\mathcal{R}=\frac{r}{1-\frac{1}{3 r}}
$$

and if $r \leq 1$,

$$
\mathcal{R}=\frac{1}{1-\frac{r}{3}}
$$

Using the relatively typical values of $p=0.6$ and $s=0.5$, one finds $\mathcal{R}=1.662$. This formula can also be used when block summing the output image. For example, a weighted block-sum of $N \times N$ pixels is equivalent to drizzling into a single pixel of size $N s$. Therefore, the correlated noise in the blocked image can be estimated by replacing $s$ with $N s$ in the above expressions. 


\section{SOME EXAMPLES OF THE APPLICATION OF DRIZZLE}

Drizzle has now been widely used for many astronomical image combination problems. In this section we briefly note some of these and provide references where further information may be obtained.

Drizzle was developed for use in the original Hubble Deep Field North, a project to image an otherwise unexceptional region of the sky to depths far beyond those of previous astronomical images. Exposures were taken in four filter bands from the near ultraviolet to the near infra-red. The resulting images are available in the published astronomical literature (Williams et al. 1996) as well as from the Space Telescope Science Institute via the World Wide Web at http://www.stsci.edu/ftp/science/hdf/hdf.html.

Subsequently Drizzle has also been applied to the Hubble Deep Field South (Williams et al. 2000). In this case it was used for the combination of images from NICMOS (Fruchter et al. 2001) and STIS (Gardner et al. 2000) as well as WFPC2 (Casertano et al. 2000). In order to obtain dithered NICMOS and WFPC2 images in parallel with STIS spectroscopy, HST was rotated, as well as shifted, between observations during the HDF-S. All the software developed to handle such challenging observations is now publicly available (see Section 9).

The HDF imaging campaigns are atypical as they had a large numbers of dither positions. A more usual circumstance, matching that described in Section 3, is the processing of a small number of dithers without multiple exposures at the same pointing. A good example of such imaging and its subsequent processing is provided in Fruchter et al. (1999) where Gamma Ray Burst host galaxies were observed using the STIS and NICMOS HST cameras to obtain morphological and photometric information. Similarly Bally, O'Dell and McCaughrean (2000) have used Drizzle to combine dithered WFPC2 images with single exposures at each dither position in a program to observe disks, microjets and wind-blown bubbles in the Orion nebula.

Examination of these published images may help the reader to obtain a feeling for the results of using the Drizzle program. In addition an extensive set of worked examples of combining dithered data using Drizzle is available in the Dither Handbook (Koekemoer et al. 2001) distributed by STScI. 


\section{CONCLUSION}

Drizzle provides a flexible, efficient means of combining dithered data which preserves photometric and astrometric accuracy, obtains optimal signal-to-noise, and approaches the best resolution that can be obtained through linear reconstruction. An extensively tested and robust implementation is freely available as an IRAF task as part of the STSDAS package and can be retrieved from the Space Telescope Science Institute web page (http://www.stsci.edu). In addition to Drizzle, a number of ancillary tasks for assisting with determining the shifts between images and the combination of WFPC2 data are available as part of the "dither" package in STSDAS.

We are continuing to improve Drizzle, to increase both ease of use and generality. New versions of Drizzle will be incorporated into STSDAS software updates. Additional capabilities will soon make the alignment of images simpler, and will provide the user with a choice of drizzling kernels, including ones designed to speed up the image combination with minimal change to the output image or weight - an enhancement which may prove particularly useful in the processing ACS images. Although these additions may make Drizzle somewhat more flexible, the basic algorithm described here will remain largely unchanged, as it provides a powerful, general algorithm for the combination of dithered undersampled images.

\section{ACKNOWLEDGEMENTS}

Drizzle was developed originally to combine the HDF-N datasets. We wish to thank our colleagues in the HDF-N team, and Bob Williams in particular, for encouraging us, and for allowing us to be part of this singularly exciting scientific endeavor. We also thank Ivo Busko for his work on the original implementation of the STSDAS dither package, Hans-Martin Adorf for many entertaining and thought provoking discussions on the theory of image combination, and Stefano Casertano for inciting us to develop a more general theory of the correlated noise in drizzled images. Finally, we are grateful to Anton Koekemoer for a careful reading of the text, and to our referee, Tod Lauer, for numerous suggestions which significantly improved the clarity and presentation of this paper. 


\section{REFERENCES}

Bally, J., O'Dell, C. R., and McCaughrean, M. J. 2000, Astron. J., 119, 2919

Bertin, E. and Arnouts, S. 1996, A\&AS, 117, 393

Casertano, S. et al. 2000, Astron. J., 120, 2747

Cowie, L. L., Hu, E. M., and Songaila, A. 1995, Astron. J., 110, 1576

Fruchter, A. S. et al. 2001, in preparation

Fruchter, A. S. et al. 1999, Astrophys. J., 516, 683

Gardner, J. P. et al. 2000, Astron. J., 119, 486

Gull, S. F. and Daniell, G. J. 1978, Nature, 272, 686

Hanisch, R. J. and White, e., R. L. 1994, The Restoration of HST Images and Spectra-II (Baltimore, STScI/NASA publication), http://www.stsci.edu/stsci/meetings/irw/

Koekemoer, A. M. et al. 2001, The HST Dither Handbook (Baltimore, STScI publication)

Lauer, T. R. 1999, Publ. Astr. Soc. Pacific, 111, 1434

Lucy, L. B. 1974, Astron. J., 79, 745

Lucy, L. B. and Hook, R. N. 1991, in Astronomical Data Analysis Software and Systems, ed. D. M. Worrall, C. Biemesderfer, and J. Barnes (Astronomical Society of the Pacific), p. 277

Richardson, B. H. 1972, J. Opt. Soc. Am., 62, 55

Stetson, P. B. 1987, Publ. Astr. Soc. Pacific, 99, 191

Storrs, A., Hook, R., Stiavelli, M., Hanley, C., and Freudling, W. 1999, NICMOS ISR-99-005, STScI

Tody, D. 1993, in Astronomical Data Analysis Software and Systems II, ed. R. J. Hanisch, R. J. V. Brissenden, and J. Barnes (A.S.P. Conference Series, Vol. 52), p. 173

Trauger, J. T. et al. 1994, Astrophys. J., 435, L3

Weir, N. and Djorgovski, S. 1990, in The Restoration of HST Images and Spectra, ed. R. L. White and R. J. Allen (STScI), p. 31 
Whitmore, B. and Heyer, I. 1997, HST Instrument Science Report WFPC2 97-08

Williams, R. E. et al. 2000, Astron. J., 120, 2735

Williams, R. E. et al. 1996, Astron. J., 112, 1335

This preprint was prepared with the AAS LATEX macros v4.0. 